\title{
Prevalence of childhood wheeze and modified DNA methylation at 7 years of age according to maternal folate levels during pregnancy in the Hokkaido Study
}

\author{
Chihiro Miyashita ${ }^{1}$, Atsuko Araki ${ }^{1}$, Ryu Miura ${ }^{1}$, Yu Ait Bamai ${ }^{1}$, Sumitaka Kobayashi $^{1}$, \\ Sachiko Itoh ${ }^{1}$, Kumiko Ito $^{2}$, Meng-Shan Tsai $^{3}$, and Reiko Kishi ${ }^{1}$ \\ ${ }^{1}$ Hokkaido University \\ ${ }^{2}$ Hokkaido University of Science \\ ${ }^{3}$ National Taiwan University
}

July 21,2020

\begin{abstract}
Background: recent epidemiological studies state that a high dose of folic acid status during pregnancy increases the risk of asthma, wheezing, and respiratory disease in childhood. As potential biological mechanism, folate acid can modify inflammation and immune susceptibility of offspring with some epigenetic differentiation, including DNA methylation. This study evaluated the association between maternal folate levels during pregnancy with childhood wheeze, and whether DNA methylation differentiation of children in genes is related to wheezing or not. Methods: the cohort comprised 6651 mother-child pairs who were evaluated for an association between maternal folate level during pregnancy and childhood wheeze at 1, 2, 4, and 7 years age, which were assessed by the International Study of Asthma and Allergies in Childhood questionnaire. Results: the median of maternal serum was $16.76 \mathrm{nmol} / \mathrm{l}$, assayed by chemiluminescent immunoassay. We found significantly increased adjusted odds ratios of childhood wheeze at 2 years age according to maternal folate levels, compared with lowest folate quartile (odds ratio $[95 \%$ confidence interval $]=$ highest; $1.27[1.03,1.56]$, and second; $1.27[1.05,1.55])$, however, no changes were observed at 1,4 , and 7 years age. In a case-control study of childhood wheeze due to DNA methylation at 7 years age, no association of maternal folate levels with DNA methylation was observed. Conclusion: our result proposes that the negative effect of maternal folate on an infant's wheeze did not remain until 7 years of age and no association with maternal folate levels and DNA methylation (at the same age) was observed.
\end{abstract}

\section{Introduction}

The World Health Organization (WHO) and most countries recommend a healthy diet plus a folic acid supplement of $400 \mu \mathrm{g} / \mathrm{d}$ from preconception until the end of the first trimester of pregnancy to seek neuraltube defects prevention (WHO, 2012). Alternatively, human studies, inconsistent annotations were observed associations between maternal folic acid intake along with folate levels during pregnancy and risk of childhood wheeze, asthma, and respiratory symptoms (Veeranki et al., 2014; Roy et al., 2018; Vereen et al., 2018). The inconsistent annotations of maternal folate can be partly explained by vary maternal folate levels and susceptibility of modified DNA methylation regarding risk of respiratory symptoms.

As potential biological mechanism, maternal folate intake can modify inflammation and immune susceptibility of offspring with some epigenetic role involving DNA methylation (Montrose et al., 2017). Folate as methyl donors change DNA methylation that is a covalent addition of a methyl group to a cytosine residue at the CpG site in the DNA sequence. In vitro experiments have suggested that epigenetic modifications also contribute to the pathogenic mechanism underlying 17q21locus, which is a potential gene related to 
asthma and harbors the adjacent genes GSDMB (Gasdermin B)/ORMDL3 (Orosomucoid-like 3) andIKZF3 (IKAROS family zinc finger 3). Recent studies have suggested that GSDMB/ORMDL3 and IKZF3 have a role in IL-6 and IL-8 levels (Wu et al., 2018), viral respiratory infections (Bouzigon et al., 2008), and childhood asthma (Breslow et al., 2010). In a Swedish study, differential DNA methylation among CpG including GSDMB/ORMDL3 andIKZF3 was associated with a risk of childhood asthma and RNA expression (Acevedo et al., 2015). However, no study evaluates whether the difference in DNA methylation in GSDMB /ORMDL3 andIKZF3 modifies the association between maternal folate level and childhood wheeze.

Participants of this study are from part of the Hokkaido Birth Cohort Study on Environment and Children's Health. The current study evaluates the associations between maternal folate levels during pregnancy and childhood wheeze and whether DNA methylation of GSDMB, ORMDL3, and IKZF3 changes the association.

\section{Materials and Methods}

\subsection{Study participants and baseline study}

In the Hokkaido Birth Cohort Study on Environment and Children's Health, the participants of 20,296 pregnant women were enrolled from 2003 to 2012 in Hokkaido, Japan. During the first trimester, participants completed the baseline questionnaire on maternal and paternal characteristics. Maternal peripheral blood samples were taken during pregnancy without fasting. Medical birth records from the delivery hospital were collected for birth weight, height, sex, and other medical conditions (Kishi et al., 2013; 2017).

The institutional ethics board for epidemiological studies at Hokkaido University Graduate School of Medicine and Hokkaido University Center for Environmental and Health Sciences approved the study protocol (approval number 69). In this study, informed consent was obtained from all study participants before enrollment.

2.2. Follow-up study until age 7 and saliva samples collection.

We have collected data regarding the wheezing symptoms of children at 1,2, 4, and 7 years of age using a modified section of the Japanese version of the International Study of Asthma and Allergies in Childhood (ISAAC) Phase Three questionnaire (Asher et al., 1995). We defined childhood wheeze as a maternal positive answer to the question "Has your child had wheezing or whistling in the chest in the past 12 months?," based on each ISAAC questionnaire administered when the child was 1, 2, 4, and 7 years of age (Figure 1). Moreover, we collected information child's history of infectious diseases (Goudarzi et al., 2018).

In a case-control study, we selected all 314 children with wheezing and randomly selected 374 control children without any allergic symptoms (extraction rate 24\%) from the ISAAC questionnaire at 7 years age. Salivary samples $(2 \mathrm{ml})$ were collected from children of case and control using The Oragene@ OG-300 DNA SelfCollection kit (DNA Genotek Inc., Ottawa, Ontario, Canada). Eventually, we used 275 salivary samples to analyze DNA methylation (Figure 1).

\subsection{Quantification of DNA Methylation}

Genomic DNA was extracted from saliva using a Maxwell 16 DNA Purification Kit (Promega, Madison, WI, USA). The DNA (500 ng) was then subjected to bisulfite conversion using Epitect Plus Bisulfite Kit (Qiagen, Venlo, The Netherlands). Bisulfite pyro sequence was performed as described previously (Murphy et al., 2012; Bollati et al., 2007). We analyzed DNA methylation in genes related to asthma for five of the CpG sites of IKZF3 (cg16293631, cg13432737), ORMDL3(cg02305874, cg14647739), and GSDMB (cg12360886). Pyrosequencing was performed using Pyromark Q24 system (Qiagen) and data were analyzed using the Pyro Q-CpG Software (Qiagen). Eventually, we completed the quantification of DNA methylation only three CpG sites of ORMDL3 (cg02305874), and IKZF3 (cg16293631, cg13432737). Other PCR primers of two CpG sites including GSDMB (cg12360886) and ORMDL3 (cg14647739) did not work. Conditions of primers were described in Supplementary Table 1. Average methylation levels of each CpG site that were analyzed in duplicate were used in statistical analyses.

2.4. Maternal folate and cotinine measurements 
Folate is quantified by direct chemiluminescent acridinium ester technology. This technique has an acceptable imprecision of less than $10.0 \%$, with an advanced Quality Control package. It has an analytical sensitivity of $0.91 \mathrm{nmol} / \mathrm{l}$. Specimen preparations, shipping, and assays were performed in batches, depending on new recruitments. We have previously reported the significance of the inverse association between maternal folate and cotinine levels (Kishi et al., 2013; Yila et al., 2012). The details of serum folate and plasma cotinine measurements are described in our previous report (Sasaki et al., 2011).

\subsection{Statistical analysis}

We analyzed the association between participant's characteristics and maternal folate level using MannWhitney's U-test and rank correlation test. In logistic regression analysis, we evaluated odds ratios (ORs) and 95\% confidence interval (CI) of childhood wheeze, according to maternal folate level, respectively. Because a biologically relevant threshold of folate on wheezing is not elucidated, we used two types of maternal folate levels as follows; first, ORs and 95\% CI for the risk of wheezing were calculated for three categorical maternal folate levels in the folate suboptimal $(6.80-13.59 \mathrm{nmol} / \mathrm{l})$ or optimal ([?] $13.60 \mathrm{nmol} / \mathrm{l}$ ) and compared to those in folate deficiency $(<6.80 \mathrm{nmol} / \mathrm{l})$ with reference to the WHO guideline (Sauberlich, 1999). Second, ORs and $95 \%$ CI for the risk of wheezing were calculated for 4 categorical maternal folate levels in the second, third, and the fourth quartiles and compared to those in the lowest quartiles as reference by the distribution of their quartiles. For the calculation of $P$ for trend, we handled categorical values of maternal folates as ordinal variables.

In a case-control study, we used two types of maternal folate levels, including categorical of quartile, and two categorical with reference to the WHO guideline, which were optimal ([?] $13.60 \mathrm{nmol} / \mathrm{l})$ or under suboptimal category $(<13.60 \mathrm{nmol} / \mathrm{l}$ ) (Supplementary Table 2$)$. In liner regression analysis, we evaluated $\beta$ and $95 \%$ CI for DNA methylation, including, three CpG sites of IKZF3 (cg16293631, cg13432737) and ORMDL3 (cg02305874), according to maternal folate levels. In logistic regression analysis, we evaluated odds ratios (ORs) and 95\% CI of childhood wheezing at 7 years age according to DNA methylation.

In both logistic and liner regression analysis, we used adjusted factors as potential confounders, including maternal age, parity, delivery year, alcohol drinking during pregnancy, log10-transformed cotinine levels, maternal allergic history, paternal allergic history, annual household income, and sex of child (model 1). Furthermore, to confounding variables of the model 1, we used adjusted factors which were collected after birth, including current maternal and paternal smoking status, breast feeding, and day care attendance (yes, no) (model 2). Pvalue of less than 0.05 was considered statistically significant. All statistical analyses were performed with SPSS software for Windows (version 21.0J; IBM, Armonk, NY, USA).

\section{Results}

In our current study, a total of 6651 participants who responded at least one of ISSAC questionnaires at 1, 2, 4, and 7 years of age were included (Figure 1). Participants were grouped by the WHO guidelines for folate deficiency are as follows: folate deficient: $\mathrm{n}=32(0.48 \%)$; suboptimal: $\mathrm{n}=1739(26.15 \%)$; and optimal: $\mathrm{n}$ $=4880(73.37 \%)$ (Table 1$)$. Associations of maternal folate levels and characteristics were shown in Table 2. We found that the adjusted OR of childhood wheeze at 2 years age according to maternal folate levels of quartiles were significantly increased, compared with that of lowest quartile, but not 1, 4, and 7 years age. Additionally, we found that adjusted $\mathrm{OR}^{2}$ of childhood wheezing at 2 years age according to maternal folate levels of WHO guideline was significantly increased trend $(P$ for trend $=0.023$ ) (Table 3).

In a case-control study, mean \pm standard deviation (SD) of DNA methylation at three CpG sites that ORMDL3 (cg02305874) andIKZF3 (cg16293631, cg13432737) are illustrated in Supplementary Table 3.We found no significant adjusted $\beta$ for DNA methylation according to maternal folate levels (Table 4). We found that adjusted OR for childhood wheezing at 7 years of age was significantly decreased with hypomethylation of IKZF3 (cg16293631), but not ORMDL3(cg02305874) and IKZF3 (cg13432737) (Supplementary Table 4). We found no significant adjusted OR for childhood wheezing at 7 years age was association maternal folate in the case-control study (Supplementary Table 4). 


\section{Discussion}

We found that adjusted OR for childhood wheezing at 2 years age according to quartile of maternal folate was significantly increased compared with that of the lowest quartile but not for 1,4 , and 7 years of age. In a case-control study of childhood wheeze for DNA methylation, which are related asthma at three CpG sites of IKZF3 and ORMDL3, no association of maternal folate with DNA methylation was noticed. This study provided novel evidence that the negative effect of maternal folate on an infant's wheezing did not remain at 7 years, and maternal folate cannot modify DNA methylation at three CpG sites ofIKZF3 and ORMDL3 of 7-year-old children.

In this study, results differ by the child's age because the infant's high sensibility to infections due to immature immune system and respiratory function. Infections are strong risk factors for infant's allergy. Furthermore, the frequency of an infant's infections until 2 years of age among wheezing children was approximately 23 fold higher than that without wheezing at 2 years of age (Supplementary Table 5). As children grow, respiratory structure and immune function mature and allergic symptoms become more stable, the negative effect of maternal folate on infant's wheezing is not observed over 4 years of age in the current study.

Medians of detected maternal serum folate levels among previous human four studies were reported as 21.5 $\mathrm{nmol} / \mathrm{l}(9.5 \mathrm{ng} / \mathrm{ml})$ in South Korea (Kim et al., 2015), $21.2 \mathrm{nmol} / \mathrm{l}(9.36 \mathrm{ng} / \mathrm{ml})$ in The Netherlands (van der Valk et al., 2013), and $51.2 \mathrm{nmol} / \mathrm{l}(22.6 \mathrm{ng} / \mathrm{ml}$ ) (Roy et al., 2018) and $43.5 \mathrm{nmol} / \mathrm{l}(19.2 \mathrm{ng} / \mathrm{ml}$ ) (Vereen et al., 2018) in the USA. Although previous studies have various sample sizes and deferent timing of maternal sample collection (first, second, or third trimester) with an outcome range of 1 to 7 years of age, these studies reported diminished or insignificant association between maternal folate levels and risk of wheezing, asthma, and respiratory symptoms in childhood. Additionally, more than $80 \%$ of participants had optimal folate of maternal intracellular folate concentration, which is a recommended biomarker for folate status than serum levels (WHO, 2012), showing decreased asthma risk at 6-7 years of age in a dose-dependent manner (Magdelijns et al., 2011). Inconsistently, in the Norwegian Study, negative effect of maternal folate (median $8.7 \mathrm{nmol} / \mathrm{l}$ ) were observed on respiratory tract infections up to 18 months, asthma at 3 and 7 years of age (Parr et al., 2017). The median maternal folate in the current study was $16.7 \mathrm{nmol} / \mathrm{l}(7.40 \mathrm{ng} / \mathrm{ml})$, which was lower than previous studies reporting a protective or null effect (Kim et al., 2015; Roy et al., 2018; van der Valk et al., 2013; Vereen et al., 2018) and higher than the Norwegian study reporting a negative effect (Parr et al., 2017). These observations signify the negative effect among the population is reasonably due to lower maternal folate levels. Therefore, negative effect at 2 years of age in this study can be caused by insufficient maternal folate status among our participants. The current study suggests that maternal folate status needs to improve.

In a case-control study, three CpG sites were not associated with maternal folate (Table 4). A previous systematic review specifies the necessity to detect modified DNA methylation change by maternal folate in the human study (Brown et al., 2014). This study is the first to report that individual DNA methylation was detected at three $\mathrm{CpG}$ sites of IKZF3 and ORMDL3 -related asthma at 7 years of age by pyrosequencing and it does not differ by maternal folate during pregnancy. Only one study has described methylation beta-values of CpG sites related to asthma, including IKZF3 and ORMDL3 using the Infinium $450 \mathrm{~K}$ assay, and asthmatic children had lower methylation than control children (Acevedo et al., 2015). In this study, same direction was observed that is inversely associated with childhood wheeze and DNA methylation of IKZF3 (Supplementary Table 4). DNA hyper-methylation of long interspersed nucleotide element -1 (LINE-1 ) from the buccal cell at 6-17 years age children, was associated with maternal intake of methyl donating nutrients including folate, but unassociated with quality of life measures among children with asthma (Montrose, 2017). LINE-1 is considered an indicator of global methylation, so dietary nutrients can affect not onlyLINE-1 but also a wide range differentiation DNA methylation among several genes. No association between maternal folate and DNA methylation at three CpG sites was observed, however, maternal folate and dietary nutrients during pregnancy can modify DNA methylation. Further study is needed to examine the modified effects of global epigenetic differentiation including the epigenome wide association study on the association between folate levels and childhood wheeze or asthma. 
The advantages of this study included the large-scale perspective birth cohort for minimum bias in the data. Furthermore, the biological assessment of folate levels to detect maternal folate in serum during pregnancy was performed. In addition, assessment of wheezing symptoms using the ISAAC questionnaire, which is an international standard questionnaire for the assessment of children's allergies was undertaken. This study had some limitations. DNA methylation for only three CpG sites were analyzed due to which global epigenetic differentiation was not covered. Also, the modified effect of DNA methylation at age 2 was not evaluated, despite its significant association with maternal folate. Finally, in this study, maternal serum folate levels were measured at a single sampling point, not intracellular folate concentration, while estimated half-life of folate was assessed at approximately $6 \mathrm{~h}$ (Sauberlich, 1999). This limitation can cause the misclassification of maternal folate levels. However, serum folate assay was commonly conducted in large epidemiological studies (Parr et al., 2017; Roy et al., 2018). Moreover, studies in Japan have reported that maternal diet habit was maintained across pregnancy (Jaw et al., 2016), stating relative stability of maternal folate levels.

\section{Funding}

The authors acknowledge all the participants and staff in this study.

This work was supported by a Grant-in-Aid for Health Science Research from the Japanese Ministry of Health, Labor and Welfare (No. 201624002B; 17932352), a Grant-in-Aid for Scientific Research from the Japanese Ministry of Education, Culture, Sports, Science and Technology (No. 25860433; 16H02645; 16K19245; 19H01071).

\section{References}

Asher, M.I., Keil, U., Anderson, H.R., Beasley, R., Crane, J., Martinez, F., et al., 1995. International study of asthma and allergies in childhood (Isaac) - rationale and methods. Eur Respir J. 8(3), 483-491.

Acevedo, N,.., Reinius, L.E., Greco, D., Gref, A., Orsmark-Pietras, C., Persson, H., Pershagen, G., Hedlin, G., Melen, E., Scheynius, A., Kere, J., Soderhall, C., 2015. 'Risk of childhood asthma is associated with CpG-site polymorphisms, regional DNA methylation and mRNA levels at the GSDMB/ORMDL3 locus', Human Mol Gen , 24, 875-890.

Bouzigon, E., Corda, E., Aschard, H., Dizier, M.H., Boland, A., Bousquet, J., et al., 2008. Effect of 17q21 variants and smoking exposure in early-onset asthma. New Engl J Med. 359(19), 1985-1994.

Breslow, D.K., Collins, S.R., Bodenmiller, B., Aebersold, R., Simons, K., Shevchenko, A., et al., 2010. Orm family proteins mediate sphingolipid homeostasis. Nature. 463(7284), 1048-U65.

Brown, S.B., Reeves, K.W., Bertone-Johnson, E.R., 2014. Maternal folate exposure in pregnancy and childhood asthma and allergy: a systematic review. Nutr Rev. 72(1), 55-64.

Bollati, V., Baccarelli, A., Hou, L., Bonzini, M., Fustinoni, S., Cavallo, D., Byun, H.M., Jiang, J., Marinelli, B., Pesatori, A.C., Bertazzi, P.A., Yang, A.S., 2007. 'Changes in DNA methylation patterns in subjects exposed to low-dose benzene'. Cancer Res . 67, 876-880.

Goudarzi, H., Konno, S., Kimura, H., Araki, A., Miyashita, C., Itoh, S., Ait Bamai, Y., Kimura, H., Shimizu, K., Suzuki, M., Ito, Y.M., Nishimura, M., Kishi, R., 2018. 'Contrasting associations of maternal smoking and pre-pregnancy BMI with wheeze and eczema in children'.Sci Total Environ. 639, 1601-1609.

Jwa, S.C., Ogawa, K., Kobayashi, M., Morisaki, N., Sago, H., Fujiwara, T., 2016. Validation of a foodfrequency questionnaire for assessing vitamin intake of Japanese women in early and late pregnancy with and without nausea and vomiting. J Nutr Sci. 5.

Kim, J.H., Jeong, K.S., Ha, E.H., Park, H., Ha, M., Hong, Y.C., Bhang, S.Y., Lee, S.J., Lee, K.Y., Lee, S.H., Kim, Y., Kim, M.H., Chang, N., 2015. 'Relationship between prenatal and postnatal exposures to folate and risks of allergic and respiratory diseases in early childhood'.Pediatr Pulmonol. 50, 155-163. 
Kishi, R., Kobayashi, S., Ikeno, T., Araki, A., Miyashita, C., Itoh, S., Sasaki, S., Okada, E., Kobayashi, S., Kashino, I., Itoh, K., Nakajima, S. Environment Members of the Hokkaido Study on, and Health Children's. 2013. 'Ten years of progress in the Hokkaido birth cohort study on environment and children's health: cohort profile-updated 2013'.Environ Health Prev Med . 18, 429-450.

Kishi, R., Araki, A., Minatoya, M., Hanaoka, T., Miyashita, C., Itoh, S., et al., 2017. The Hokkaido birth cohort study on environment and children's health: cohort profile-updated 2017. Environ Health Prev Med. $22(1)$.

Magdelijns, F.J., Mommers, M., Penders, J., Smits, L., Thijs, C., 2011. Folic acid use in pregnancy and the development of atopy, asthma, and lung function in childhood. Pediatrics. 128(1), e135-e144.

Mallol, J., Crane, J., von Mutius, E., Odhiambo, J., Keil, U., Stewart, A., et al., 2013. The International Study of Asthma and Allergies in Childhood (ISAAC) Phase Three: A global synthesis. Allergol Immunopathol (Madr). 41(2), 73-85.

Montrose, L., Ward, T.J., Semmens, E.O., Cho, Y.H., Brown, B., Noonan, C.W., 2017. 'Dietary intake is associated with respiratory health outcomes and DNA methylation in children with asthma'. Allergy Asthma Clin Immunol. 13, 12.

Murphy, S.K., Adigun, A., Huang, Z., Overcash, F., Wang, F., Jirtle, R.L., Schildkraut, J.M., Murtha, A.P., Iversen, E.S., Hoyo, C., 2012. 'Gender-specific methylation differences in relation to prenatal exposure to cigarette smoke'. Gene . 494, 36-43.

Parr, C.L., Magnus, M.C., Karlstad, O., Haugen, M., Refsum, H., Ueland, P.M., McCann, A., Nafstad, P., Haberg, S.E., Nystad, W., London, S.J., 2017. Maternal folate intake during pregnancy and childhood asthma in a population-based cohort. Am J Respir Crit Care Med . 195, 221-228.

Rosas-Salazar, C., Hartert, T.V., 2017. 'Prenatal exposures and the development of childhood wheezing illnesses'. Curr Opin Allergy Clinical Immunol. 17, 110-115.

Roy, A., Kocak, M., Hartman, T.J., Vereen, S., Adgent, M., Piyathilake, C., Tylavsky, F.A., Carroll, K.N., 2018. Association of prenatal folate status with early childhood wheeze and atopic dermatitis. Pediatr Allerg Immunol. 29, 144-150.

Sasaki, S., Braimoh, T.S., Yila, T.A., Yoshioka, E., Kishi, R., 2011. Self-reported tobacco smoke exposure and plasma cotinine levels during pregnancy-a validation study in Northern Japan. Sci Total Environ . 412-413, 114-118.

Sauberlich, H.E., 1999. Folate (FA, pteroylmonoglutamic acid, folacin). In: Laboratory Tests for the Assessment of Nutritional Status, 2nd ed., pp. 103-134 [I Wolinsky, editor]. Washington, DC: CRC Press.

van der Valk, R.J., Kiefte-de Jong, J.C., Sonnenschein-van der Voort, A.M., Duijts, L., Hafkamp-de Groen, E., Moll, H.A., Tiemeier, H., Steegers, E.A., Hofman, A., Jaddoe, V.W., de Jongste, J.C., 2013. 'Neonatal folate, homocysteine, vitamin B12 levels and methylenetetrahydrofolate reductase variants in childhood asthma and eczema'. Allergy , 68, 788-795.

Veeranki, S.P., Gebretsadik, T., Dorris, S.L., Mitchel, E.F., Hartert, T.V., Cooper, W.O., Tylavsky, F.A., Dupont, W., Hartman, T.J., Carroll, K.N., 2014. Association of folic acid supplementation during pregnancy and infant bronchiolitis'. AmJ Epidemiol. 179, 938-946.

Veeranki, S. P., Gebretsadik, T., Mitchel, E.F., Tylavsky, F.A., Hartert, T.V., Cooper, W.O., Dupont, W.D., Dorris, S.L., Hartman, T.J., Carroll, K.N, 2015. Maternal folic acid supplementation during pregnancy and early childhood asthma'. Epidemiology . 26, 934-941.

Vereen, S., Gebretsadik, T., Johnson, N., Hartman, T.J., Veeranki, S.P., Piyathilake, C., Mitchel, E.F., Kocak, M., Cooper, W.O., Dupont, W.D., Tylavsky, F., Carroll, K.N., 2018. Association between maternal 2nd trimester plasma folate levels and infant bronchiolitis'. Matern Child Health J . 23, 164-172. 
World Health Organization (WHO) - 2012. Guideline: daily iron and folic acid supplementation in pregnant women. ISBN-13: 978-924-150199-6.https://www.ncbi.nlm.nih.gov/books/NBK132263/

Wu, X.L., Li, R., Zhang, H.W., Jin, R., Wang, J.Y., Juan, C.X., et al., 2018. Methylation status of ORMDL3 regulates cytokine production and p-ERK/MMP9 pathway expression. Exp Cell Res. 372(1), 43-51.

Yila, T.A., Araki, A., Sasaki, S., Miyashita, C., Itoh, K., Ikeno, T., Yoshioka, E., Kobayashi, S., Goudarzi, H., Baba, T., Braimoh, T., Minakami, H., Endo, T., Sengoku, K., Kishi, R., 2016. Predictors of folate status among pregnant Japanese women: the Hokkaido Study on Environment and Children's Health, 2002-2012'. Br J Nutr. 115: 2227-2235.

Zetstra-van der Woude, P.A., De Walle, H.E., Hoek, A., Bos, H.J., Boezen, H.M., Koppelman, G.H., de Jong-van den Berg, L.T., Scholtens, S., 2014. 'Maternal high-dose folic acid during pregnancy and asthma medication in the offspring'. Pharmacoepidemiol Drug Safe. 23, 1059-1065.

\section{Hosted file}

200721_Tables.docx available at https://authorea.com/users/344723/articles/471144-prevalenceof-childhood-wheeze-and-modified-dna-methylation-at-7-years-of-age-according-tomaternal-folate-levels-during-pregnancy-in-the-hokkaido-study

\section{Hosted file}

200629_Figures.docx available at https://authorea.com/users/344723/articles/471144prevalence-of-childhood-wheeze-and-modified-dna-methylation-at-7-years-of-age-accordingto-maternal-folate-levels-during-pregnancy-in-the-hokkaido-study 